\title{
Um conhecimento local sobre o uso de plantas medicinais em Lumiar, Nova Friburgo, RJ
}

\author{
A local knowledge on the use of medicinal plants in \\ Lumiar, Nova Friburgo, RJ
}

Un conocimento local sobre el uso de plantas

medicinales em Lumiar, Nova Friburgo, RJ

\begin{tabular}{r}
\hline Revista Dissertar No32 V.1 ANO XV \\
DOI: 10.24119/16760867ed115269 \\
Data de submissão: 19-04-2019 \\
Data de aceite: $01-05-2019$
\end{tabular}

por Valéria Costa Rocha Viana ${ }^{1}$

\section{Resumo}

A biodiversidade das florestas tropicais tem reconhecida importância e investigações etnofarmacológicas e etnobotânicas têm sido usadas como estratégia de seleção de plantas medicinais reconhecidas por cientistas em todo o mundo. Esta pesquisa objetivou investigar o uso informal de plantas medicinais em Lumiar, Nova Friburgo, RJ. Tratase de um estudo descritivo, baseado em dados coletados pela aplicação de entrevistas abertas e semi-estruturadas entre janeiro e outubro de 2018. Nova Friburgo, RJ possui ocorrência de vegetação exuberante que reúne atributos atrativos, principalmente Lumiar, $5^{\circ}$ Distrito, que a partir da década de 80 sofreu uma invasão turística. A partir daí, os espaços rurais foram substituídos por espaços de lazer e o local saiu do seu isolamento parcial, o que acarretou em grandes mudanças no estilo de vida de seus moradores. Os dados coletados serviram para revelar os modos como as plantas medicinais são utilizadas, suas partes e indicações articulado ao contexto cultural do qual faz parte. PALAVRAS-CHAVE: Plantas medicinais. Etnofarmacologia. Etnobotânica

\footnotetext{
Abstract

Tropical forests biodiversity has been recognized and ethnopharmacological and ethnobotanical investigations have been used as

1 Valéria Costa Rocha Viana: Possui graduação em Farmácia pela Universidade Federal Fluminense (1997), especialização em Homeopatia pelo Instituto Hahnemanianno do Brasil (2004) e mestrado em Ciência Ambiental, linha de pesquisa Gestão Ambiental, pela Universidade Federal Fluminense (2010). É doutoranda em Ciências Para Produtos Aplicados a Saúde (PPG-CAPS UFF) desde julho de 2019. Atualmente é professora na Universidade Estácio de Sá (UNESA), em Nova Friburgo. valeriaviana08@hotmail.com
} 
108 Um conhecimento local sobre o uso de plantas medicinais em Lumiar, Nova Friburgo, RJ

a strategy for the selection of medicinal plants recognized by scientists around the world. The objective of this research was to investigate the informal use of medicinal plants in Lumiar, Nova Friburgo, RJ. It is a descriptive study, based on data collected through the application of open and semi-structured interviews between January and October, 2018. Nova Friburgo, RJ has exuberant vegetation that meets attractive attributes, mainly in Lumiar, 5th District, which on the 80s suffered a tourist invasion. From there, the rural spaces were replaced by spaces of recreation and the place left its partial isolation, which entailed in great changes in the lifestyle of its residents. The collected data served to reveal the ways in which medicinal plants are used, their parts and indications articulated to the cultural context of which they are part. KEYWORDS: Medicinal plants. Ethno-pharmacology. Ethnobotanic.

\section{Reumen}

La biodiversidad de los bosques tropicales há reconocido importância e investigaciones etnofarmacológicas y etnobotánica han sido usadas como estrategia de selección de plantas medicinales reconocidas por científicos em todo el mundo. Esta investigación objetivó reconocer el uso informal de plantas medicinales en Lumiar, Nova Friburgo, RJ. Se trata de un studio descriptivo, basado en datos recolectados por La aplicación de entrevistas abiertas y estructuradas entre enero y octubre de 2018. Nova Friburgo, RJ posee ocurrencia de vegetación exuberante que reune atributos atractivos, principalmente Lumiar, $5^{\circ}$ Distrito a partir de la década de los 80 sufrió uma invasão turística. A partir de ahí los espacios rurales fueron substituidos por espacios de ocio y el local salió de su aislamiento, lo que acarreó en grandes câmbios en el estilo de vida de SUS habitantes. Los datos recolectados sirvieron para revelar los modos como lãs plantas medicinales son utilizadas, SUS partes e indicaciones articuladas al contexto cultural Del cual forma parte.

PALABRAS CLAVE: Plantas medicinales. Ethnopharmacology. Etnobotánica.

\section{Introdução}

O futuro da humanidade passa pela sustentabilidade. $\mathrm{O}$ conceito de sustentabilidade não é uma exclusividade ambiental, pois perpassa por vários âmbitos, incluindo a sustentabilidade política, econômica, social, cultural, tecnológica e também na saúde, entre outros.

Estima-se que o Brasil possua $22 \%$ das plantas conhecidas no mundo, um patrimônio que vem sendo alvo da cobiça por parte de 
países do primeiro mundo (SHARAPIN, 2000). É considerado um dos países com maior diversidade vegetal, abrigando 55 mil espécies catalogadas, sendo que 4 mil dessas espécies vegetais são utilizadas para fins medicinais, resultado da observação e manejo da flora por povos tradicionais (ZUCCHI, 2009). Segundo Sachs (2002) não é preciso retroceder no tempo para entender o potencial da natureza, mas sim transformar o conhecimento apreendido ao longo dos anos da história.

Entre as razões que justificam o interesse crescente pelas plantas medicinais, nos países industrializados, estão a escassez de novas descobertas, pelos processos tradicionais de síntese química, de moléculas farmacologicamente ativas e passíveis de uso em terapêutica, os efeitos colaterais decorrentes do uso correto ou abusivo de fármacos sintéticos, e a mudança do perfil do consumidor que, desde o final da década de 80 , do século XX, tem preferido produtos naturais no lugar dos sintéticos, em vários setores como a saúde, alimentação, vestuário ou higiene (SHARAPIN, 2000), sem contar o equilíbrio do meio ambiente envolvido nessa questão.

A Mata Atlântica é um dos biomas mais importantes e mais ricos em biodiversidade do planeta, entretanto, é um dos mais ameaçados e muitas espécies de plantas estão em extinção (SOS MATA ATLÂNTICA, 2012). O Estado do Rio de Janeiro é um lugar onde se pode notar a presença de remanescentes deste bioma, principalmente na região serrana, com ocorrência de vegetação exuberante, rios, cachoeiras e clima de serra, que reúne atributos bastante atrativos para um tipo de turismo que possibilite um contato com a natureza.

Particularmente no caso do $5^{\circ}$ Distrito, Lumiar, que a partir da década de 80, com o asfaltamento da estrada que o liga a Mury, essa localidade começou a perder sua característica de isolamento parcial, mantida desde os tempos da colonização e começou a sofrer uma "invasão" de turistas. Então, os espaços rurais, originalmente destinados à lavoura, foram substituídos por espaços de lazer, provocados pela atividade turística (CARNEIRO, 2000). Até aquele momento, sua população era composta basicamente por agricultores que mantinham muito pouco ou nenhum contato com o meio urbano (TEIXEIRA, 1998). Dessa maneira, muitas modificações em diversos âmbitos passaram a acontecer sem que se tenha tido tempo de se dar conta de quanta coisa podia estar sendo transformada naquele processo.

A biodiversidade presente nas florestas tropicais tem reconhecida importância ecológica, econômica, social e cultural, mas o conhecimento científico do seu potencial ainda é restrito, o que demonstra que pesquisas podem subsidiar trabalhos sobre usos sustentáveis e apontar para potenciais da biodiversidade através 
110 Um conhecimento local sobre o uso de plantas medicinais em Lumiar, Nova Friburgo, RJ

da valorização e do aproveitamento do conhecimento empírico das comunidades inseridas nesse ambiente (QUINTEIRO, 2008).

O uso de plantas no tratamento e na cura de enfermidades é tão antigo quanto à espécie humana, simbolizando muitas vezes o único recurso terapêutico de muitas comunidades e grupos étnicos (MACIEL et al., 2002). Porém, essa prática vem sendo ameaçada por diversos fatores como a facilidade de acesso à medicina moderna e a saída das pessoas de seus ambientes naturais para regiões urbanas, levando assim à perda do conhecimento popular herdado e transferido há várias gerações (PINTO et al., 2006).

A falta de médicos disponíveis e o acesso difícil a medicamentos permitiram a abertura de espaços sociais ocupados por práticas terapêuticas populares (PUMAR-CANTINI, 2005) que faziam com que pessoas recorressem freqüentemente àqueles que detinham o conhecimento das propriedades medicinais das plantas: os erveiros, ou até mesmo os rezadeiros. Essa era uma prática que fazia parte do cotidiano da comunidade de Lumiar, que pouco a pouco foi perdendo seu espaço com a chegada de outros recursos advindos do desenvolvimento, que no caso do $5^{\circ}$ Distrito de Nova Friburgo, pode ser compreendido pela urbanização sofrida lá.

As investigações etnofarmacológicas e etnobotânicas têm sido usadas como estratégia de seleção de plantas medicinais reconhecidas por cientistas em todo o mundo. A grande contribuição de muitos estudos é apresentar uma lista de espécies (animais ou plantas) com informações sobre seus usos por comunidades locais. Tais pesquisas continuam resgatando o período inicial das investigações etnocientíficas, fortemente carregadas de uma influência etnográfica (ALBUQUERQUE, 2005).

Essa biodiversidade associada a uma rica diversidade étnica e cultural detém um valioso conhecimento tradicional associado ao uso de plantas medicinais, e possui o potencial necessário para desenvolvimento de pesquisas com resultados em tecnologias e terapêuticas apropriadas (BRASIL, 2006). É necessário que se faça uma abordagem holística e interdisciplinar, onde cientistas trabalhem juntos na construção de caminhos para uso e aproveitamento dos recursos da natureza de maneira sábia, que respeite a sua diversidade. Uma boa combinação de recursos naturais abundantes e baratos, aliado à força de trabalho qualificado e conhecimento, podem resultar em vantagem competitiva inigualável (SACHS, 2002).

Para Geertz (1989), quando se quer compreender o que é ciência, não se deve olhar, em primeiro lugar, para suas teorias ou descobertas, mas sim, para o que os praticantes da ciência fazem.

Nesse sentido, algumas perguntas poderiam ser feitas. Que plantas são utilizadas nas práticas de cura utilizadas no cotidiano 
dessas pessoas? Como as mudanças sociais afetam esse saber e sua transmissão? O que há em comum entre seus praticantes?

Por conta disso, o estudo de determinados aspectos do saber passado a várias gerações, principalmente aquele passado através da história oral, torna-se importante para registrá-lo antes que possa se perder definitivamente.

Na busca pela compreensão das relações que permeiam o uso de plantas medicinais, as pesquisas etnodirigidas têm demonstrado ser poderosas ferramentas. Aponta-se aqui como objetivo geral investigar sobre o uso e o conhecimento informal, o saber-fazer, de plantas medicinais por parte dos erveiros / rezadeiros de Lumiar, Nova Friburgo, RJ. Na direção de contextualizar o estudo, objetivouse ainda relatar como é orientado o uso de plantas medicinais pelos detentores desse saber local (indicações, formas de preparo, partes usadas das plantas, coleta).

\section{A área de estudo e a coleta de dados}

A presente pesquisa trata-se de um estudo descritivo baseado em dados coletados que apresentou como roteiro a aplicação de entrevistas abertas e semi-estruturadas. Na abordagem qualitativa torna-se importante a utilização de um roteiro que sirva como facilitador e orientador em uma conversa (JORGE \& MORAIS, 2002).

Para registro dos dados a opção de primeira escolha foi a gravação, por possibilitar a captação das informações com maior riqueza de detalhes, que não se faria possível através do registro escrito.

Para compor o tamanho da amostra foram eleitos alguns critérios de inclusão para participação no estudo tais como: pessoas que moram no local há mais de quarenta anos, que sejam conhecedores da paisagem local e das plantas medicinais e que por vezes orientam seu uso a outras pessoas da comunidade. De acordo com esses critérios esse é um número reduzido de representantes, o que faz da presente pesquisa um estudo de caso. Os atores locais são chamados de erveiros ou rezadeiros. A definição da categoria erveiros seguiu de acordo com as realizadas por Quinteiro (2008) e Loyola (1984) que afirmam serem estes, especialistas em ervas naturais, além de darem consultas, fazerem diagnósticos e prescreverem tratamentos. $\mathrm{O}$ rezador, por outro lado, cura através de meios simbólicos.

A coleta de dados se deu no período de março a outubro de 2018. Foram aplicadas as técnicas de "informação cruzada"- submeter a um informante dados fornecidos por outro informante, para confirmação ou refutação dos mesmos, e de informação repetida - indagar ao mesmo informante as mesmas perguntas para aumento no grau de confiança das respostas, como mencionado por Albuquerque e Lucena (2004). 
112 Um conhecimento local sobre o uso de plantas medicinais em Lumiar, Nova Friburgo, RJ

Todos os colaboradores formalizaram sua participação na pesquisa através da assinatura de um Termo de Consentimento Livre e Esclarecido (TCLE) e a pesquisa cujo CAAE possui $\mathrm{n}^{\circ}$ 79581117.4.0000.5284 foi aprovada pelo CEP da UNESA/RJ através do Parecer Consubstanciado $\mathrm{n}^{\circ}$ 2.571.658 de março de 2018.

Situado numa área montanhosa, o município de Nova Friburgo faz parte do bioma Mata Atlântica, na região serrana fluminense, a uma altitude de $846 \mathrm{~m}$, possui clima tropical de altitude. Ocupa uma área de $938,5 \mathrm{~km}^{2}$, correspondentes a 13,5\% da área da Região Serrana. Compreende os distritos de Nova Friburgo, Riograndina, Campo do Coelho, Amparo, Conselheiro Paulino, Lumiar, São Pedro da Serra e Mury (TRIBUNAL DE CONTAS DO ESTADO, 2008).

Fazendo parte da Área de Proteção Ambiental (APA) Macaé de Cima, a área de estudo compreende Lumiar, o $5^{\circ}$ Distrito de Nova Friburgo, RJ, o maior do município. Possui uma área de 397 km², desde a cabeceira do Rio Macaé, em Macaé de Cima, até as fronteiras com Casimiro de Abreu e Bom Jardim. A localidade possui em média 600 $m$ de altitude (PRÓ-MEMÓRIA, 2009). O pequeno povoado de Lumiar dista cerca de $170 \mathrm{~km}$ da cidade do Rio de Janeiro. O local abriga diversificada fauna e flora e suas muitas cachoeiras possuem águas límpidas e abundantes.

Lumiar apresenta um povoamento disperso pela sua extensa zona rural, o que faz com que os moradores dessa zona predominem sobre a urbana, que embora em menor número, foi a que mais cresceu (CARNEIRO, 1998) no recorte temporal desde a década de 80 do sec. XX. Atualmente conta com uma população de cerca de 5.000 pessoas (TRIBUNAL DE CONTAS DO ESTADO, 2011).

\section{Plantas medicinais e medicina popular}

O uso de plantas medicinais é milenar e com o passar do tempo tem ocorrido um crescente interesse pelo estudo de espécies vegetais e seu uso tradicional em diferentes partes do mundo (CHEIKHYOUSSEF et al., 2011). De acordo com Ibiapina et al. (2014), um dos marcos históricos importantes sobre a utilização de plantas medicinais no mundo foi a Declaração de Alma Ata em 1978, onde foi reconhecido o uso de plantas medicinais e de fitoterápicos com finalidade profilática, curativa e paliativa. Desde então a Organização Mundial da Saúde (OMS) passou a reconhecer as plantas medicinais e a Fitoterapia.

A prática de se utilizar os medicamentos fitoterápicos vem crescendo devido a este significar uma terapia mais complementar ao organismo, por apresentar poucos efeitos adversos e também por se tratar de medicamentos com baixo custo (BRUNING et al., 2012).

Utilizada como medicina alternativa complementar, a 
fitoterapia pode revelar importante funcionalidade. A classificação de fitoterápico é dada como, produto do processamento de plantas medicinais, de uso tradicional na população, capaz de prevenir, aliviar ou curar doenças (CARVALHO et al., 2007).

Para milhões de pessoas, as plantas, os tratamentos tradicionais e as práticas da medicina tradicional representam a principal fonte de atenção à saúde e às vezes a única (ORGANIZAÇÃ̂ MUNDIAL DA SAÚDE, 2013).

Muitas pesquisas etnobotânicas têm se ocupado muito em registrar as plantas, seus usos e indicações terapêuticas, deixando de lado as questões sócio-culturais, imprescindíveis nesse tipo de abordagem (ALBUQUERQUE \& LUCENA, 2004).

A etnografia deve ser capaz de revelar um mundo subjetivo e subentendido para dar significado ao objeto pesquisado dentro de um determinado contexto. De acordo com Geertz (1989) uma das coisas que o etnógrafo tem a fazer, muitas vezes, é lidar com conceitos complexos, com estruturas estranhas ou inexplícitas e que ele tem que, de alguma forma, primeiro aprender e depois apresentar. Dessa maneira o pesquisador deve conhecer a comunidade e a população que estão sendo estudadas a fim de contextualizar o objeto de pesquisa e dar o sentido etnográfico à mesma.

\section{Os atores locais encontrados e seus percursos etnográficos}

Foram entrevistados cinco atores locais, que são também os detentores de um saber local. No presente trabalho entende-se saber local como o acervo de conhecimento empírico acerca das plantas e suas propriedades medicinais, bem como alguns procedimentos de cura ou até mesmo de coleta dessas plantas que envolvem, ou não, componentes místico-religiosos. Essas pessoas são conhecidas na comunidade como aquelas que dominam o uso das plantas medicinais nas práticas de cura.

Para consolidar a compreensão da relação entre magia e medicina, há que se admitir que o simbólico imponha uma série de significações que a objetividade causal e racional não pode explicar (MARTINS, 2003).

A automedicação é particularmente preocupante quando é realizada em conjunto com outros medicamentos, podendo levar a efeitos sinérgicos e interações não esperadas pelo médico (VEIGA JUNIOR, 2008). A chamada medicina popular pode ser definida como um conjunto de técnicas de tratamento empregadas pelos especialistas não reconhecidos pela medicina oficial. $\mathrm{O}$ conhecimento e as técnicas da medicina popular são vistas como sobrevivências folclóricas de uma época passada, de regiões rurais e comunidades tradicionais isoladas 
114 Um conhecimento local sobre o uso de plantas medicinais em Lumiar, Nova Friburgo, RJ

e atrasadas, que vão desaparecendo frente ao desenvolvimento da urbanização (LOYOLA, 1984).

É justamente nesses especialistas que reside o objetivo maior deste trabalho. Essas pessoas já não são encontradas com facilidade na área de estudo indicando que as mudanças sócio-ambientais e culturais ocorridas, principalmente a urbanização que o local vem sofrendo, são fatores que interferem nessa prática que configura numa das representações sociais que passa a ocupar um lugar discreto na vida das pessoas desse lugar.

Novas tecnologias e modificações trazidas pelo homem em ambientes naturais fazem com que a medicina popular seja cada vez menos utilizada pela sociedade (PIRES et al., 2009). As intensas transformações pelas quais Lumiar passou imprimiram grandes mudanças naidentidade social, cultural, econômica, política e ambiental de seus moradores. Principalmente pela grande urbanização sofrida e pela transformação do Distrito em Ârea de Proteção Ambiental, duas características que catalisaram a vinda de muitas pessoas de fora e foram responsáveis pela intensa urbanização que aconteceu por lá.

Rezadeiros foram indagados a respeito de suas práticas e verificou-se que alguns não se utilizam de plantas em suas rezas. Outros possuem amplo conhecimento das plantas e também preparam alguns remédios caseiros. Uma das entrevistadas prepara xaropes somente para as pessoas da família, segundo ela, e o faz utilizando sempre "sete ou nove plantas", que podem variar entre si, de acordo com a disponibilidade em se obter cada uma. Ela diz que os números, sete ou nove, são escolhidos por serem ímpares, sem maiores explicações. Completa que uma das plantas utilizadas só pode ser colhida se crescer sobre plantas que não possuem espinho, para não provocar "fisgadas" [sic] pelo corpo de quem tomar o remédio feito com essa erva, o que revela um elemento mágico nessa prática.

Outra pessoa entrevistada fabrica xaropes e chás para distribuir a quem precisar. Não cobra pelos seus serviços, diz que faz "por prazer e pelo amor ao próximo, para ajudar aos necessitados". Também reza se for preciso e suas fórmulas variam de acordo com a disponibilidade de se encontrar as plantas, não havendo precisão nem rigor nelas. Porém, deixa clara a preferência em se utilizar plantas frescas ao invés de secas, não fazendo nenhuma restrição quanto à época ou hora da colheita.

Outro erveiro quando entrevistado também menciona a reza como um dos componentes utilizados em suas curas. Possui muitas plantas medicinais cultivadas na sua casa e outras que sabe localizar na região, baseado num mapeamento mental feito por ele mesmo. Comenta que para cada doença existem várias plantas com capacidade de curá-la e as utiliza em sua maior parte na forma de chás, mas para 
alguns casos utiliza também emplastros feitos com essas plantas. Acredita que ele "tem essa missão a cumprir, que foi designada por Deus" e diz "que todos têm uma tarefa para cumprir na Terra e que cada um tem que fazer a sua parte".

De acordo com alguns autores, o universo da religião faz parte de um sistema simbólico no qual os indivíduos ancoram suas práticas. Segundo Pumar-Cantini (2005) a fé em alguma entidade maior que sustenta e mantém a saúde e a harmonia de todos é o denominador comum que conecta o conjunto de saberes e práticas desses indivíduos. Esse saber ocupa seu espaço na comunidade estudada e aponta para um mesmo aspecto encontrado por essa autora.

De acordo com Jorge e Morais (2002), a abordagem da doença é feita, não em um esquema biomecanicista, mas sim cósmico, onde a percepção sobre a doença e sua cura se baseia na visão cosmológica da comunidade em estudo e certas peculiaridades relacionadas ao emprego de plantas medicinais não podem ser entendidas se não forem levados em consideração os fatores culturais envolvidos e o ambiente físico em que ocorre.

Outro erveiro entrevistado possui um arsenal dessas ervas, plantadas num jardim na frente de sua casa. Sua prática também é carregada de misticismo, manifestando-se até na coleta de algumas espécies, como uma chamada mal-com-tudo que relata que "se a pessoa sair à procura não a encontra, mas se falar mal com tudo, bem comigo, acha". Novamente o elemento místico se revela. Essa característica mística se encontra presente entre todos os entrevistados, permitindose traçar um perfil semelhante entre esses atores locais, mesmo entre aqueles que não praticam a reza. Outro aspecto que merece destaque é sua fala sobre as mudanças ocorridas no local desde que a área estudada se transformou em APA:

Aqui tinha uma fartura imensa! $O$ pessoal aqui nem comprava, todo mundo dava. Aqueles que não tinha, ganhava [sic]. Eu me lembro que eu ganhava. Hoje, se a senhora, só vê casa caída e o pessoal mudaram [sic] tudo pra cidade. É tudo de gente, só tem graúdo de fora, só tem proibida a entrada, proibido a caçada, proibido a pescada, proibida a passagem. Se a senhora ver, não vê mais um pé de inhame, não vê mais um pé de banana, não vê mais um pé de planta, não vê mais nada. É tudo a poder de mercado. Gente que tinha terreiro cheio de vaca, galinha, porco, fartura numerosa! Couve, lavoura, muita coisa! Hoje, se quiser um franguinho, tem que comprar no mercado.

Para ilustrar o percurso etnográfico, outro entrevistado, filho 
116 Um conhecimento local sobre o uso de plantas medicinais em Lumiar, Nova Friburgo, RJ

de um curandeiro e rezador ao mesmo tempo, não seguiu essa carreira, que afirma "ser de muita responsabilidade", lembrando das noites em que seu pai saía de casa com chuva para atender "os necessitados de suas curas". Porém, orgulhoso, dá uma receita para mordidas de cobra e diz que "hoje em dia muito pouco novo sabe alguma coisa". Uma de suas filhas trabalha com plantas medicinais de uma maneira diferente das praticadas na região até o presente momento: ela comercializa plantas secas na forma de chás. O trabalho é realizado junto com um grupo de mulheres, que funciona como se fosse uma cooperativa, porém não formalizada. Seu registro é de produtora rural, mas todas as decisões são tomadas em grupo e o lucro é igualmente dividido. São comercializadas cinqüenta espécies, todas da região, segundo ela. Algumas são cultivadas e outras são de ocorrência espontânea. Esse fato é comentado por Carneiro (2009) que diz que com a melhoria das vias de comunicação, além da modificação dos espaços de sociabilidade, a produção mercantil vai ganhando espaço na roça familiar. O que se vê aqui é que além de ocupar espaço na roça familiar, essa prática mercantil aparece relacionada ao uso das plantas medicinais pautada na apropriação de um saber local que pode, ou não, ajudar na sua consolidação, mas que aponta para uma forma diferente das dos atores locais em se relacionar com esse saber.

Todos os entrevistados aprenderam a usar as plantas com seus antepassados, através da vivência e da sua aplicação no cotidiano. Esse resultado também foi compartilhado nos estudos de Arnous e colaboradores (2005) e de Grandi e colaboradores (1989) quando pesquisaram o uso de plantas medicinais em outras comunidades.

\section{O modo local de uso das plantas encontradas}

Foram citadas 61 plantas medicinais, suas partes usadas, indicações e formas de preparo. No presente trabalho o nome das plantas não foi revelado por não ter sido realizada a identificação botânica das espécies. Grandi e colaboradores (1989) destacam que é perigoso promover o uso de plantas medicinais somente pelo nome vulgar porque este varia muito de uma região para outra e mesmo numa determinada região uma mesma espécie pode receber vários nomes. O mesmo aspecto é apontado por França e colaboradores (2008) que acrescentam ainda que os princípios ativos podem variar em função de condições climáticas, de solo, época do ano, entre outros. Além disso, muitas pesquisas etnobotânicas têm se ocupado muito em registrar as plantas, seus usos e indicações terapêuticas, deixando de lado as questões sócio-culturais, imprescindíveis nesse tipo de abordagem como apontaram Albuquerque e Lucena (2004).

Em relação às partes utilizadas das plantas, verificou-se que as 
folhas representam a maioria, seguidas das flores, caules e frutos. Raízes, bulbos e sementes também foram citados, embora em menor número (Figura 1). As plantas também foram utilizadas preferencialmente no estado fresco.

Figura 1: Principais partes usadas das plantas citadas

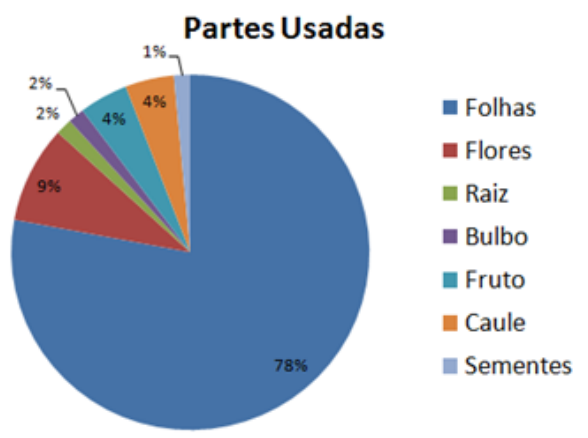

Quanto à forma de preparo o chá ganhou destaque, seguido de banhos, xaropes e em menor número, foram citados a infusão com álcool, sumo, entre outras. Em estudo realizado por Arnous e colaboradores (2005) o preparo na forma de chá também foi o mais apontado, ao pesquisarem plantas medicinais de uso caseiro no município de Datas, MG. Para os autores o aproveitamento adequado dos princípios ativos contidos nas plantas depende do correto modo de preparo levando-se em consideração as partes dos vegetais que são utilizadas.

Figura 2: Formas de preparo das plantas medicinais

\section{Formas de Preparo}

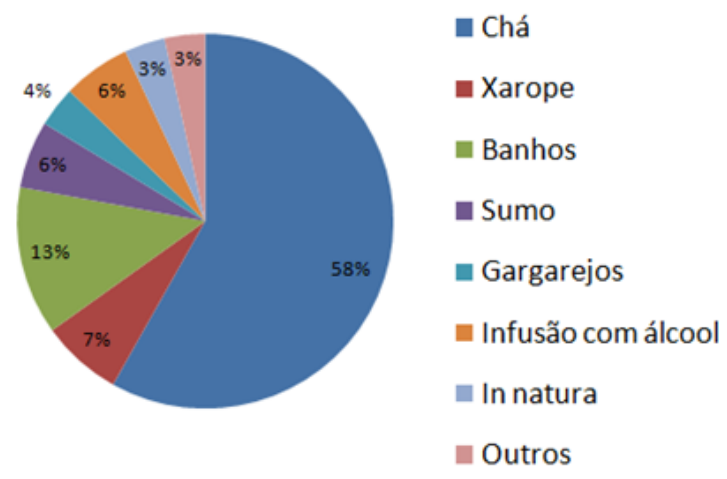

Entre os problemas de saúde que são tratados com plantas, os mesmos foram classificados de acordo com o CID 10 (Classificação 
118 Um conhecimento local sobre o uso de plantas medicinais em Lumiar, Nova Friburgo, RJ

Estatística Internacional de Doenças e Problemas Relacionados com a Saúde).

Entre os sintomas e sinais gerais, os mais citados foram febre e dor de cabeça. Já entre os problemas do aparelho digestivo, pode-se dizer que enjôo, diarréia e vômito ganharam destaque, mas também os problemas referentes ao estômago e fígado não deixaram de ser apontados. Entre as doenças do aparelho respiratório, o destaque fica para a gripe, doença que geralmente acomete as vias respiratórias superiores. Entre as doenças do aparelho geniturinário, os problemas renais e as cólicas menstruais, não deixaram de ser citados. Também foram citados dores musculares e nas articulações, que ficaram enquadradas nas doenças do sistema osteomuscular e do tecido conjuntivo, seguidas das doenças do sistema nervoso, onde as plantas medicinais são utilizadas como calmante, em sua maioria. Por fim, também se registrou o uso dessas plantas para o tratamento de vermes, algumas doenças infecciosas e parasitárias, e do coração, órgão nobre que se encaixa nas doenças do aparelho circulatório. Esses usos apontam para uma idéia de que as plantas não fazem mal por se tratarem de produtos naturais (Figura 3 ).

Figura 3: As doenças mais citadas no tratamento com plantas

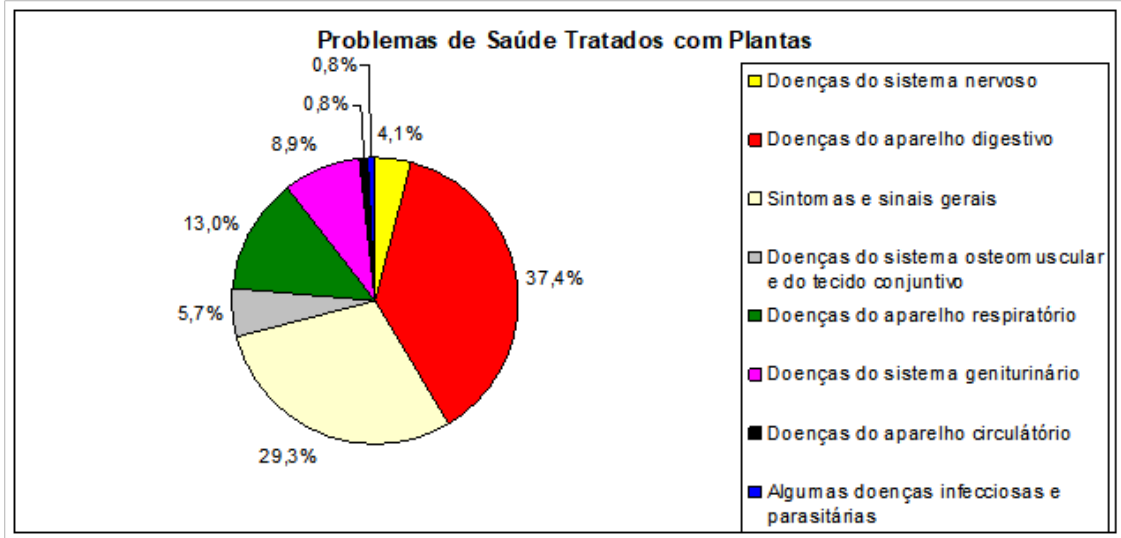

Em estudo realizado por França e colaboradores (2008) foi observado que seus entrevistados compartilhavam desta idéia e que algumas indicações se encontravam em desacordo com a literatura. A crença na naturalidade das plantas medicinais não é facilmente contestada, pois as evidências científicas de ocorrência de intoxicações e efeitos colaterais relacionados com o uso destas plantas consistem em informações que dificilmente chegam ao alcance dos usuários (SILVEIRA et al., 2008). Além disso, ao contrário do que ocorre com outros medicamentos, na fitoterapia as pessoas encontram um 
método de cura e prevenção mais acessível, porém isso não exclui a importância da orientação de um profissional de saúde quanto ao uso indiscriminado de algumas plantas (BRUNING et al., 2012).

As formas de uso das plantas relatadas e suas indicações neste estudo apontam para uma semelhança de percepções que foram apontadas por outros autores, indicando a mesma visão de que plantas só trazem benefícios à saúde sem efeitos colaterais na maioria das vezes.

\section{Considerações finais}

$\mathrm{O}$ aspecto mais marcante que pôde ser observado na presente pesquisa foi a forma como suas práticas e seu saber estão alicerçados em crenças que motivam e norteiam essas práticas. É como se elas formassem um cimento em torno do qual, seu saber está consolidado e fortalecido. Foi percebida a existência de um rico acervo de conhecimento e de uma grande plasticidade em relação aos usos das plantas, cercados de misticismo e de uma aura de sobrenatural. Seu saber é herança, ou resultado, de um modo de vida diferente do que hoje se dá no local. Preservar o conhecimento/cultura da população local e seu modo de vida faz parte da preservação de um patrimônio, porém imaterial, que está interligado com o potencial e o aproveitamento da biodiversidade. Portanto, está totalmente inserido na questão ambiental. Apesar da área de estudo ter sido transformada em APA, sofreu intensa urbanização e esse aspecto ficou deixado sem a devida importância com a qual poderia ter sido tratada.

Apesar da melhoria ao acesso aos serviços de saúde, com a presença de Postos, de médicos e de farmácias, a demanda por esses serviços ainda é muito maior que a oferta, o que deixa o espaço disponível a ser ocupado por práticas e conhecimentos populares relacionados a esse contexto.

Os poucos atores locais encontrados não estão repassando seu conhecimento para as gerações futuras e seu registro tornase fundamental para que não se perca definitivamente. $O$ presente estudo deixa apontado muitas possibilidades de direções para outras possíveis pesquisas, tão necessárias quando o assunto é o homem e sua forma de relacionar-se com o meio ambiente que o cerca, matéria fértil que requer diferentes olhares para um assunto que está longe de ser esgotado.

\section{Agradecimentos}

Esse trabalho foi possível graças ao apoio financeiro fornecido pela 
120 Um conhecimento local sobre o uso de plantas medicinais em Lumiar, Nova Friburgo, RJ

Diretoria de Pesquisa Aplicada - DPA - da UNESA.

Referências

ALBUQUERQUE, U. P.; LUCENA, R. F. P. Métodos e técnicas na pesquisa etnobotânica. Recife: Editora Livro Rápido, 2004. 189 p.

ALBUQUERQUE, U. P. Introdução a Etnobotânica. $2^{\mathrm{a}}$ ed. Rio de Janeiro: Editora Interciência, 2005. 80 p.

ARNOUS, A. H.; SANTOS, A. S.; BEINNER, R. P. C. Plantas medicinais de uso caseiro - conhecimento popular e interesse por cultivo comunitário. Revista Espaço para a Saúde, Londrina, v. 6, n. 2, p. 1-6, jun. 2005. Disponível em: < https://s3.amazonaws.com/ academia.edu.documents/32856432/ plantamedicinal.pdf?AWSAcce ssKeyId=AKIAIWOWYYGZ2Y53UL3A\&Expires=1540076916\&Sign ature $=$ TYbkhov19On5QDvw4Ur2WYUowfI\%3D\&response-contentdisposition=inline $\% 3 \mathrm{~B} \%$ 20filename $\% 3$ DPLANTAS_MEDICINAIS_ DE_USO_CASEIRO_-_CONH.pdf> Acesso em: 20 out 2018.

BRASIL. Ministério da Saúde. Portaria 971, maio de 2006. Aprova as Práticas Integrativas e complementares. Brasília: Ministério da Saúde, 2006. Disponível em: <http://bvsms.saude.gov.br/bvs/publicacoes/ pnpic.pdf> Acesso em: 01 set 2018.

BRUNING, M, C, R; MOSEGUI, G, B, G; VIANNA, C, M, M; 2012. A utilização da fitoterapia e de plantas medicinais em unidades básicas de saúde nos municípios de Cascavel e Foz do Iguaçu - Paraná: a visão dos profissionais de saúde. Ciência \& Saúde e Coletiva, p. 2675-2685, 2012.

CARNEIRO, M. J. Memória, Esquecimento e Etnicidade na Transmissão do Patrimônio Familiar. In: CARNEIRO, M. J.; GIULIANI, G. M.; MEDEIROS, L. S. de \& RIBEIRO, A. M. M. (orgs.). Campo Aberto, o rural no Estado do Rio de Janeiro. Rio de Janeiro: Contra Capa Livraria Ltda., 1998. 334 p. p 273-291.

. Descendentes de Suíços e Alemães de Nova Friburgo: de "Colonos" a "Jardineiros da Natureza". In: GOMES, A. de C. (org.). Histórias de Imigrantes e de Imigração no Rio de Janeiro. Rio de Janeiro: 7 Letras, 2000. 206 p. p 44-65.

. De "produtor" a "consumidor": Mudanças sociais e hábitos alimentares. In: NEVES, D. P. Processos de constituição e reprodução do campesinato no Brasil. São Paulo: Editora UNESP; Brasília, DF: Núcleo de Estudos Agrários e Desenvolvimento Rural, 
2009. 326 p. v. 2. (História social do campesinato brasileiro).

CARVALHO, J. A. de; SANTOS, C. S. S.; CARVALHO, M. P. de; SOUZA, L. S. de. O alimento como remédio: considerações sobre o uso de alimentos funcionais. Rev. Cient. ITPAC. Araguaína. V. 6. $\mathrm{N}^{\mathrm{o}}$ 4. Out. 2013. Disponivel em: <https://assets.itpac.br/arquivos/ Revista/64/1.pdf>. Acesso em: 26 out 2018.

CHEIKHYOUSSEF, A.; SHAPI, M.; MATENGU, K.; ASHEKELLE, H. $\mathrm{M}$. Ethnobotanical study of indigenous knowledgeon medical plant use by traditional healers in Oshikoto region, Namibia. Journal of Ethnobiology and Etnomedicine, v. 7, n.10, p. 1-11, 2011. Disponível em: < https://www.ncbi.nlm.nih.gov/pmc/articles/PMC3062575/> Acesso em: 25 ago 2018.

FRANÇA, I. S. X de; SOUZA, J. A. de; BAPTISTA, R. S.; BRITTO, V. R. de S. Medicina popular: benefícios e malefícios das plantas medicinais. Rev. Bras. Enferm., Brasilia, 2008, mar-abr: 61(2): 201-8. Disponível em: <http://www.redalyc.org/html/2670/267019607010/> Acesso em: 20 out 2018

GEERTZ, C. A Interpretação das Culturas. Rio de Janeiro: LTC - Livros Técnicos e Científicos S. A., 1989. 215 p.

GRANDI, T. S. M.; TRINDADE, J. A. da; PINTO, M. J. F.; FERREIRA, L. L.; CATELLA A. C. Plantas medicinais de Minas Gerais, Brasil. Acta Bot. Bras., Feira de Santana , v. 3, n. 2, supl. 1, p. 185-224, 1989 Disponível em: <http://www.scielo.br/scielo.php?script=sci_ arttext\&pid=S0102-33061989000300018\&lng=en\&nrm=iso>. Acesso em: 20 out 2018.

IBIAPINA, W.V.; LEITÃO, B.P.; BATISTA, M.M.; PINTO, D. S. Inserção da Fitoterapia na atenção primária aos usuários do SUS. Rev. Ciência Saúde Nova Esperança. Jun, 12(1): p.58-68, 2014. Disponível em: < http://www.facene.com.br/wp-content/ uploads / 2010/11/INSER \% C3\% 87\% C3\% 83O-DA-FITOTERAPIANA - A T EN \% C 3 \% $87 \%$ C3 \% 83 O-PRIM \% C3 \% 81 RIA - A OS USU\%C3\%81RIOS-DO-SUS.pdf> Acesso em: 25 ago 2018.

JORGE, S. S. A.; MORAIS, R. G. Etnobotânica de Plantas Medicinais. ANAIS DO I SEMINÁRIO MATO-GROSSENSE DE ETNOBIOLOGIA E ETNOECOLOGIA \& IISEMINÁRIO CENTRO-OESTE DE PLANTAS MEDICINAIS, 2003, Cuiabá. In: COELHO, M. F. B.; JUNIOR, P. C.; DOMBROSKI, J. L. D. (org.). Diversos Olhares em Etnobiologia, 
122 Um conhecimento local sobre o uso de plantas medicinais em Lumiar, Nova Friburgo, RJ

Etnoecologia e Plantas Medicinais. Cuiabá - MT: UNICEN Publicações, 2002. 250 p. v. 1. p. 89-98.

LOYOLA, M. A. Médicos e Curandeiros - conflito social e saúde. São Paulo: DIFEL, 1984. 198 p.

MACIEL, M. P. M.; PINTO, A. C.; VEIGA JÚNIOR., V. F. Plantas Medicinais: A Necessidade de Estudos Multidisciplinares. Química Nova, São Paulo, v. 25, n. 3, p. 429-438, maio. 2002

MARTINS, P. H. Contra a desumanização da medicina: crítica sociológica das práticas médicas modernas. Petrópolis: Vozes, 2003. $335 \mathrm{p}$.

ORGANIZAÇÃO MUNDIAL DA SAÚDE. Estrategia de La OMS sobre medicina tradicional - 2014-2023. Disponível em: <http://apps.who. int/iris/bitstream/10665/95008/1/9789243506098_spa.pdf> Acesso em: 01 de set 2018 .

PINTO, E. de.P.P; AMOROZO, M. C. de. M;FURLAN, A. Conhecimento popular sobre plantas medicinais em comunidades rurais de mata atlântica- Itacaré, BA, Brasil. Revista Acta Botanica Brasilica, v.20, n.4, p. 751-762, 2006. Disponível em: <http://www.scielo.br/pdf/\%0D/ abb/v20n4/01.pdf> Acesso em: 16 maio 2018.

PIRES, M. V.; ABREU, P. P.; SOARES, C. S.; SOUZA, B.; MARIANO, D.; SILVA, D. da C.; ROCHA, E. A. Etnobotânica nos terreiros de candomblé nos municípios de Ilhéus e Itabuna, Bahia, Brasil. Revista Brasileira de Biociências, Porto Alegre, v.7, n.1, p. 3-8, jan./mar. 2009. Disponível em: < http://www.ufrgs.br/seerbio/ojs/index.php/rbb/ article/viewFile/1108/839> Acesso em: 19 out 2018

PUMAR-CANTINI, L. Prática Curativa: Um Saber Sonegado? Rio de Janeiro, 2003. 122 f. Dissertação (Mestrado em História das Ciências da Saúde) - Casa de Oswaldo Cruz, Fiocruz, Rio de Janeiro. 2005.

QUINTEIRO, M. M. C. Etnobotânica aplicada à definição de estratégias de conservação em Visconde de Mauá, Área de Proteção Ambiental da Serra da Mantiqueira. Niterói, 2008, 177 f. Dissertação (Mestrado em Ciência Ambiental) - Instituto de Geociências, Universidade Federal Fluminense, Niterói. 2008.

SACHS, I. 2002. Caminhos para o desenvolvimento sustentável. $4^{\text {a }}$ ed. 
Rio de Janeiro: Garamond. 96 p.

SILVEIRA, Patricia Fernandes; BANDEIRA, Mary Anne Medeiros; ARRAIS, Paulo Sérgio Dourado. Farmacovigilância e reações adversas às plantas medicinais e fi toterápicos: uma realidade. Revista Brasileira de Farmacognosia , Fortaleza, p. 1-9, out. 2008. Disponível em: <http:/ / www.scielo.br/pdf/rbfar/v18n4/v18n4a21>. Acesso em: 11 nov 2018.

SOS MATA ATLÂNTICA. A Mata Atlântica. Disponível em: <http:/ / www.sosmatatlantica.org.br>. Acesso em: 16 mai 2018.

TEIXEIRA, V. L. Pluriatividade e Agricultura Familiar na Região Serrana do Estado do Rio de Janeiro. Rio de Janeiro, 1998, 185 f. Dissertação (Mestrado em Desenvolvimento, Agricultura e Sociedade) - Universidade Federal Rural do Rio de Janeiro, Rio de Janeiro. 1998.

TRIBUNAL DE CONTAS DO ESTADO. ESTUDOS SOCIOECONÔMICOS DOS MUNICÍPIOS DO ESTADO DO RIO DE JANEIRO 2008 . 2008. Disponível em: <http:/ / file:/ / D:/Patrick\%20 Galv\%C3\%A3o/Downloads/Estudo\% 20Socioecon \%C3\%B4mico\%20 2008\%20-\%20Nova\%20Friburgo.pdf>. Acesso em: 16 mai 2018.

TRIBUNAL DE CONTAS DO ESTADO. ESTUDOS SOCIOECONÔMICOS DOS MUNICÍPIOS DO ESTADO DO RIO DE JANEIRO 2011. Disponível em: <https://www.tce.rj.gov.br/ documents/10180/1092020/Estudo\%20Socioecon \% C3\% B4mico \% 20 2011\%20-\%20Nova\%20Friburgo.pdf> Acesso em: 20 out 2018.

VEIGA JUNIOR, V. F. Estudo do consumo de plantas medicinais na Região Centro-Norte do Estado do Rio de Janeiro: aceitação pelos profissionais de saúde e modo de uso pela população. Revista Brasileira de Farmacognosia, João Pessoa, v. 18, n. 2, p. 308-313. Abr/Jun, 2008.

ZUCCHI, M.I. Diversidade genética em espécies medicinais. In: IX JORNADA PAULISTA DE PLANTAS MEDICINAIS. SÃO PAULO, SP, BRASIL. 2009. Disponível em: <http://www.infobibos.com/ artigos/2009_4/diversidadegenetica/index.htm> Acesso em: 16 mai 2018. 
RDISSERTARDISSERTARDISSERTARDISSHRTARDISSERTARDISSERTARDISL

TARDISSERTARDISSERTARDISSERTARDISSERTARDISSERTARDISSERTARD HRTARDISSERTARDISSERTARDISSERTARDISSERTARDISSERTARDISSERTA] SSERTARDISSERTARDISSERTARDISSERTARDISSERTARDISSERTARDISSER RDISSERTARDISSERTARDISSERTARDISSERTARDISSERTARDISSERTARDISE TARDISSERTARDISSERTARDISSERTARDISSERTARDISSERTARDISSERTARD HRTARDISSERTARDISSERTARDISSERTARDISSERTARDISSERTARDISSERTA] SSERTARDISSERTARDISSERTARDISSERTARDISSERTARDISSERTARDISSER RDISSERTARDISSERTARDISSERTARDISSERTARDISSERTARDISSERTARDISC TARDISSERTARDISSERTARDISSERTARDISSERTARDISSERTARDISSERTARD HRTARDISSERTARDISSERTARDISSERTARDISSERTARDISSERTARDISSERTA] SSERTARDISSERTARDISSERTARDISSERTARDISSERTARDISSERTARDISSER RDISSERTARDISSERTARDISSERTARDISSERTARDISSERTARDISSERTARDISE TARDISSERTARDISSERTARDISSERTARDISSERTARDISSERTARDISSERTARD RTARDISSERTARDISSERTARDISSERTARDISSERTARDISSERTARDISSERTA] SSERTARDISSERTARDISSERTARDISSERTARDISSERTARDISSERTARDISSER RDISSERTARDISSERTARDISSERTARDISSERTARDISSERTARDISSERTARDISE TARDISSERTARDISSERTARDISSERTARDISSERTARDISSERTARDISSERTARD HTARDISSERTARDISSERTARDISSERTARDISSERTARDISSERTARDISSERTA] SSERTARDISSERTARDISSERTARDISSERTARDISSERTARDISSERTARDISSER RDISSERTARDISSERTARDISSERTARDISSERTARDISSERTARDISSERTARDISE TARDISSERTARDISSERTARDISSERTARDISSERTARDISSERTARDISSERTARD HRTARDISSERTARDISSERTARDISSERTARDISSERTARDISSERTARDISSERTA] SSERTARDISSERTARDISSERTARDISSERTARDISSERTARDISSERTARDISSER RDISSERTARDISSERTARDISSERTARDISSERTARDISSERTARDISSERTARDISE TARDISSERTARDISSERTARDISSERTARDISSERTARDISSERTARDISSERTARD HRTARDISSERTARDISSERTARDISSERTARDISSERTARDISSERTARDISSERTA] SSERTARDISSERTARDISSERTARDISSERTARDISSERTARDISSERTARDISSER RDISSERTARDISSERTARDISSERTARDISSERTARDISSERTARDISSERTARDISC 「ARDISSERTARDISSERTARDISSERTARDISSERTARDISSERTARDISSERTARD HRTARDISSERTARDISSERTARDISSERTARDISSERTARDISSERTARDISSERTA] SSERTARDISSERTARDISSERTARDISSERTARDISSERTARDISSERTARDISSER RDISSERTARDISSERTARDISSERTARDISSERTARDISSERTARDISSERTARDISE 「ARDISSERTARDISSERTARDISSERTARDISSERTARDISSERTARDISSERTARD HRTARDISSERTARDISSERTARDISSERTARDISSERTARDISSERTARDISSERTA] SSERTARDISSERTARDISSERTARDISSERTARDISSERTARDISSERTARDISSER RDISSERTARDISSERTARDISSERTARDISSERTARDISSERTARDISSERTARDISE 「ARDISSERTARDISSERTARDISSERTARDISSERTARDISSERTARDISSERTARD HRTARDISSERTARDISSERTARDISSERTARDISSERTARDISSERTARDISSERTA] SSERTARDISSERTARDISSERTARDISSERTARDISSERTARDISSERTARDISSER RDISSERTARDISSERTARDISSERTARDISSERTARDISSERTARDISSERTARDISE TARDISSERTARDISSERTARDISSERTARDISSERTARDISSERTARDISSERTARD HRTARDISSERTARDISSERTARRTARDISSERTARDISSERTARDISSERTARDISS IARDISSERTARDISSERTARDISSERTARDISSERTARDISSERTARRTARDISSER RDISSERTARDISSERTARDISSERTARDISSERTARDISSERTARDISSERTARDISE 